\title{
RANDOM FOURIER TRANSFORMS
}

\author{
BY \\ G. A. HUNT
}

Paley and Zygmund [10]( $\left.{ }^{1}\right)$ have shown that for almost every random choice of signs the series

$$
\sum \pm a_{n} e^{i n t}
$$

represents a continuous function, provided only that

$$
\sum a_{n}^{2}(\lg n)^{1+\epsilon}<\infty .
$$

A little later B. Jessen [5] studied random almost periodic functions. One of his theorems, stated in the language of probability, is this: Let $\lambda_{1}, \lambda_{2}, \ldots$ be real and let $Y_{1}, Y_{2}, \cdots$ be independent complex random variables, each $Y_{n}$ being uniformly distributed on the circumference $|y|=1$. If

$$
\sum a_{n}^{2} e^{\sigma\left|\lambda_{n}\right|}<\infty
$$

for some $\sigma>0$, then almost certainly

$$
\sum a_{n} Y_{n} e^{i \lambda_{n} t}=o(\lg t)^{1 / 2},
$$$$
t \rightarrow \infty \text {. }
$$

It is our purpose to show that the $\pm a_{n}$ or the $a_{n} Y_{n}$ may be replaced by any independent random variables $X_{1}, X_{2}, \cdots$ subject to $\varepsilon\left\{X_{n}\right\}=0$ and $\sum \varepsilon\left\{X_{n}^{2}\right\}<\infty$. (Here $\varepsilon\{X\}$ denotes the expectation of $X$.) We shall define

$$
Z(t)=\sum X_{n} e^{i \lambda_{n} t}
$$

and prove that if

$$
\sum \varepsilon\left\{X_{n}^{2}\right\}\left\{\lg \left(1+\left|\lambda_{n}\right|\right)\right\}^{1+e}<\infty,
$$

then almost certainly $Z(t)$ is continuous in $t$; that if

$$
\sum \varepsilon\left\{X_{n}^{2}\right\}\left|\lambda_{n}\right|^{2 \alpha}<\infty
$$

for some positive $\alpha \leqq 1$, then almost certainly

$$
Z(t)=o(\lg t)^{1 / 2},
$$$$
t \rightarrow \infty,
$$

and moreover $Z(t)$ has modulus of continuity of the form $K h^{\alpha}(\lg 1 / h)^{1 / 2}$. More precise statements will be found in Theorems 2 ( $\$ 1), 4(\$ 5), 7(\$ 8)$.

The proofs are based on two inequalities which are of interest in themselves. They are Lemma 1 ( $\$ 2)$ and Lemma 10 (\$6). Luckily both can be ex-

Received by the editors August 22, 1950.

(1) Numbers in brackets refer to the references at the end of the paper. 
tended to integrals and give us the means of dealing with $\int e^{i \lambda t} X(d \lambda)$. This integral is defined in $\S 4$; its continuity is studied in $\$ 4$ and $\S 5$, its mean behavior in $\$ 7$, and its growth in $\$ 8$.

In $\$ 10$ we specialize $X(d \lambda)$ to obtain the Fourier-Wiener transform (which is apparently the only example of interest). It is interesting to note that Theorem 12 contains a family of statements, one of which is the usual law of the iterated logarithm for Brownian motion $(\beta=0$; the finer theorems, say those in Feller [4], escape our analysis). In the same way, Theorem 11 contains the local law of the iterated logarithm, and Theorem 13 the uniform Hölder condition.

Proofs are given in full only through $\$ 4$, for the details of later proofs are much the same.

I have bent the arguments as far as possible into the form given by Paley-Zygmund and Jessen. Their papers contain a good many results other than those above which can be generalized by using Lemmas 1 and 10 . Paley and Zygmund, however, have another set of theorems-roughly, that $\sum \pm a_{n} \exp \left(i \lambda_{n} t\right)$ behaves badly if the $a_{n}$ behave badly. Most of these can be generalized to random variables satisfying

$$
\mathcal{E}\left\{\left|X_{n}\right|^{1+\epsilon}\right\} \leqq A\left\{\varepsilon\left|X_{n}\right|\right\}^{1+\epsilon}
$$

for some constant $A$. The results are not of much interest except to provide counter examples-and these are furnished in abundance by $\pm a_{n}$.

One has only to glance to Jessen's paper and those of Paley and Zygmund to see how greatly I am indebted to them. Of quite as much help were many conversations with $\mathrm{S}$. Bochner.

1. Two theorems on series. We suppose that $\Omega$ is a set with elements $\omega$; that $B$ is a Borel field of sets $E$ contained in $\Omega$; that $P(E)$ is a measure defined on $\mathcal{B}$; that $\Omega \in \mathcal{B}$; and that $P(\Omega)=1$. A random variable is any complex-valued function defined on $\Omega$ and measurable $\mathcal{B}$. We write $d \omega$ for the element of measure in the integral, thus-

$$
\int_{E} X(\omega) d \omega \equiv \int_{E} X(\omega) P(d E)
$$

Until $\S 4$ we deal with the series $\sum X_{n}(\omega) e^{i \lambda_{n} t}$. Here the $\lambda_{n}$ are real numbers, and the random variables $X_{n}(\omega)$ are subject to three conditions:

$\left(\mathrm{A}_{1}\right) X_{1}, X_{2}, \cdots$ are independent;

(A $\mathrm{A}_{2} \int_{\Omega} X_{n}(\omega) d \omega=0, n=1,2, \cdots$;

$\left(\mathrm{A}_{3}\right) B=\sum_{n=1}^{\infty} b_{n}=\sum_{n=1}^{\infty} \int_{\Omega}\left|X_{n}\right|^{p} d \omega<\infty$.

The last condition need hold only for one value of $p, 1 \leqq p \leqq 2$.

Let us define

$$
Z_{n}(t, \omega)=\sum_{k=1}^{n} X_{k}(\omega) e^{i \lambda_{k} t}
$$




$$
Z(t, \omega)=\lim _{n \rightarrow \infty} Z_{n}(t, \omega) .
$$

Kolmogorov's three series theorem implies that the limit exists almost everywhere on the space $\Omega \times(t)$, if on this space the measure is the product of $d \omega$ and ordinary Lebesgue measure $d t$.

Our goal in the next two sections is to establish the following theorems:

TheOREM 1. For almost all $\omega \in \Omega$ and for every $\rho$,

$$
\int_{-\infty}^{\infty} A_{\rho}\left\{Z_{*}(t, \omega)\right\} \frac{d t}{1+t^{2}}<\infty .
$$

Here we have used the notation

$$
\begin{aligned}
Z_{*}(t, \omega) & =\sup _{1 \leqq n<\infty}\left|Z_{n}(t, \omega)\right|, \\
A_{\rho}\{u\} & =e^{\rho|u|^{2}}-1 .
\end{aligned}
$$

TheOREM 2. Suppose that $1<\lambda_{1}<\lambda_{2}<\cdots$ and that

$$
\sum_{1}^{\infty} b_{n}\left(\lg \lambda_{n}\right)^{1+\epsilon}<\infty
$$

for some $\epsilon>0$. Then, for almost all $\omega \in \Omega$,

$$
\frac{Z_{n}(t, \omega)}{1+t^{2}} \rightarrow \frac{Z(t, \omega)}{1+t^{2}}
$$$$
n \rightarrow \infty,
$$

uniformly in $t \in(-\infty, \infty)$. In particular, $Z(t, \omega)$ is continuous in $t$ for almost all $\omega$.

The hypothesis that the $\lambda_{n}$ increase is unnecessary in Theorem 2; the statement as it stands, however, is just what we need in $\$ 4$ when we consider random integrals. We prove in $\$ 2$ an inequality (Lemma 1), from which Theorem 1 follows at once. The proof of Theorem 2, which is an adaptation of one of Paley-Zygmund, is carried out in $\$ 3$.

2. An inequality.

Lemma 1. Let $\rho$ be given. Then there is a function $\psi=\psi_{\rho}(\omega)$ which is everywhere greater than zero and has the property that

$$
\int_{\Omega} A_{\rho}\left\{\sup _{n}\left|\sum_{1}^{n} c_{k} X_{k}(\omega)\right|\right\} \psi(\omega) d \omega \leqq 1
$$

for every sequence $c_{1}, c_{2}, \cdots$ satisfying $\left|c_{k}\right| \leqq 1$.

The important fact is that $\psi$ does not depend on the sequence $c_{k}$; it does depend, of course, on $\rho$ and on the $X_{n}$. The proof is long. We break it up into 
a number of lemmas.

A random variable $X$ is symmetric if $P\{X \in G\}=P\{-X \in G\}$ for every open set $G$.

Lemma 2. Let $U_{1}, \cdots, U_{n}$ be independent and symmetric. Let $S_{k}=U_{1}$ $+\cdots+U_{k}, S_{*}=\sup _{k}\left|S_{k}\right|$. Then

$$
\int_{\Omega} S^{p} d \omega \leqq 2 \int_{\Omega}\left|S_{n}\right|^{p} d \omega
$$

The inequality, a special case of one contained in Marcinkiewicz-Zygmund [8], can be proved quite simply. Suppose the $U_{k}$ are real. For any $A>0$ let $E=E_{A}$ be the set on which $\sup _{k} S_{k} \geqq A$ and $E_{k}$ the set on which $S_{1}<A, \cdots$, $S_{k-1}<A, S_{k} \geqq A$. The $E_{k}$ are disjoint and $E=U E_{k}$. Since $U_{k+1}+\cdots+U_{n}$ is symmetric and independent of $E_{k}$, there is a subset $F_{k}$ of $E_{k}$ satisfying " $P\left(F_{k}\right) \geqq P\left(E_{k}\right) / 2$ " and " $U_{k+1}+\cdots+U_{n} \geqq 0$ if $\omega \in F_{k}$." The $F_{k}$ are disjoint and $S_{n} \geqq A$ on $F_{k}$. Hence

$$
P\left\{\sup _{\mathbf{k}} S_{k} \geqq A\right\}=\sum P\left(E_{k}\right) \leqq 2 \sum P\left\{F_{k}\right\} \leqq 2 P\left\{S_{n} \geqq A\right\} .
$$

By symmetry, $P\left\{\inf _{k} S_{k} \leqq-A\right\} \leqq 2 P\left\{S_{n} \leqq-A\right\}$, so that $P\left\{S_{*} \geqq A\right\}$ $\leqq 2 P\left\{\left|S_{n}\right| \geqq A\right\}$. If the right member of (9) is finite, then $P\left\{S_{*} \geqq A\right\}$ $\leqq 2 P\left\{\left|S_{n}\right| \geqq A\right\}=o\left(A^{-p}\right)$ and the following integrations by parts are justified:

$$
\begin{gathered}
\int S_{*}^{p} d \omega=-\int_{0}^{\infty} A^{p} d P\left\{S_{*} \geqq A\right\}=\int_{0}^{\infty} P\left\{S_{*} \geqq A\right\} d A^{r} \\
\leqq 2 \int_{0}^{\infty} P\left\{\left|S_{n}\right| \geqq A\right\} d A^{p}=2 \int\left|S_{n}\right|^{p} d \omega .
\end{gathered}
$$

If the $U_{k}$ are complex, (9) is proved in much the same way.

Since we shall often consider suprema let us write

$$
[a]_{*}=\sup _{m}\left|\sum_{k=1}^{m} a_{k}\right| \text {. }
$$

Also define $\mu_{q}$ to be the $2 q$ th moment of a Gaussian variable:

$$
\mu_{q}=\frac{1}{(2 \pi)^{1 / 2}} \int_{-\infty}^{\infty} u^{2 q} e^{-u^{2} / 2} d u=1 \cdot 3 \cdot 5 \cdots(2 q-1) .
$$

Lemma 3. Suppose that $U_{1}, \cdots, U_{n}$ are symmetric and independent, that $\left|U_{k}\right| \leqq 1$, and that $\sum_{1}^{n}\left|a_{k}\right|^{2}=1$. Let $S=\sum_{1}^{n} a_{k} U_{k}$. Then

$$
\int_{\Omega}|S|^{2 q} d \omega \leqq \mu_{q}
$$


This is essentially Khintchine's inequality [6]. When $|S|^{2 q}$ is expanded, a typical term has the form

$$
\prod_{1}^{n} a_{k}^{\alpha_{k}} \bar{a}_{k}^{\beta_{k}} U_{k}^{\alpha_{k}} \bar{U}_{k}^{\beta_{k}}
$$

Since the $U_{k}$ are symmetric and independent the expectation of this term is zero unless all $\alpha_{k}+\beta_{k}$ are even. In any case, the expectation is not greater than $\prod\left|a_{k}\right|^{\alpha_{k}+\beta_{k}}$ in absolute value. Let us compare $S$ with $T=\sum\left|a_{k}\right| Y_{k}$, where the $Y_{k}$ are independent Gaussian variables. In the expansion of $T^{2 q}$ the term corresponding to (13) is $\prod\left|a_{k}\right|^{\alpha_{k}+\beta_{k}} Y_{k}^{\alpha k+\beta k}$. Its expectation is zero unless all $\alpha_{k}+\beta_{k}$ are even. In any case its expectation is as great as that of (13). Now $T$ is itself a normalized Gaussian variable, for $\sum\left|a_{k}\right|^{2}=1$. Hence (12) is true.

Combining Lemmas 2 and 3 we have the following lemma.

LEMMA 4. If the $U_{k}$ are independent and symmetric, if $\left|U_{k}\right| \leqq 1$, and if $\sum\left|a_{k}\right|^{2}=M$, then

$$
\int_{\Omega}[a U]_{*}^{2 q} d \omega \leqq 2 \mu_{q} M^{q}
$$

Lemma 5 (Kolmogorov [7]). If $W_{1}, W_{2}, \cdots$ are independent, if $\int W_{k} d \omega$ $=0$, and if $H=\sum \int\left|W_{k}\right|^{2} d \omega$, then

$$
P\left\{[W]_{*} \leqq K H^{1 / 2}\right\} \geqq 1-1 / K^{2} .
$$

We are now ready to begin the proof proper of Lemma 1. Until Lemra $8^{\prime}$ we assume that $p=2$ in $\left(A_{3}\right)$.

Lemma 6. Suppose that the $X_{k}$ are symmetric, that $p=2$, and that $M>0$. Define $E \subset \Omega$ by

$$
\sum_{1}^{\infty}\left|X_{k}(\omega)\right|^{2}<M
$$

Then, for every sequence $c_{k}$ with $\left|c_{k}\right| \leqq 1$,

$$
\int_{E}[c X]_{*}^{2 q} d \omega \leqq 2 \mu_{q} M^{q}, \quad q=1,2, \cdots
$$

The truth of the lemma clearly depends only on the distribution of the $X_{k}$. This being so, we take $\Omega^{\prime}=(0<u<1) \times \Omega$ and $X_{n}^{\prime}\left(\omega^{\prime}\right)=\phi_{n}(u) X_{n}(\omega)$, with $\phi_{n}(u)$ the $n$th Rademacher function. Let $E^{\prime}=(0<u<1) \times E$. The $\phi_{n}(u)$ on $E^{\prime}$ satisfy the hypotheses of Lemma 4 ; so for every $\omega \in E$

$$
\int_{0}^{1}[c \phi(u) X(\omega)]_{*}^{2 q} d u \leqq 2 \mu_{q} M^{q} .
$$


Integrate over $E$ :

$$
\int_{E^{\prime}}\left[c X^{\prime}\left(\omega^{\prime}\right)\right]_{*}^{2 q} d \omega^{\prime}=\int_{E} d \omega \int_{0}^{1}[c \phi(u) X(\omega)]_{*}^{2 q} d u \leqq 2 \mu_{q} M^{q} .
$$

Since the $X_{n}^{\prime}$ are distributed on $\Omega^{\prime}$ as the $X_{n}$ on $\Omega$, this last inequality proves the theorem.

We remove the restriction that the $X_{n}$ be symmetric.

Lemma 7. Suppose that $p=2$, that $M>4 B\left(\right.$ see $\left.\left(\mathrm{A}_{3}\right)\right)$, and define $E$ again by (14). Then, for every sequence $\left|c_{k}\right| \leqq 1$,

$$
\int_{E}[c X]_{*}^{2 q} d \omega \leqq 4 \mu_{q}(9 M)^{q}, \quad q=1,2, \cdots
$$

Let $\Omega^{\prime}, E^{\prime}, P^{\prime}, X_{n}^{\prime}\left(\omega^{\prime}\right)$ be replicas of $\Omega, E, P, X_{n}(\omega)$. The variables $Y_{n}=X_{n}$ $-X_{n}^{\prime}$, considered on the space $\Omega \times \Omega^{\prime}$ (which is provided with product measure) are independent and symmetric. On $E \times E^{\prime}, \sum\left|Y_{n}\right|^{2} \leqq 4 M$. Thus, according to Lemma 6 ,

$$
\int_{E} \int_{E^{\prime}}[c Y]_{*}^{2 q} d \omega d \omega^{\prime} \leqq 2 \mu_{q}(4 M)^{q}
$$

Let $F^{\prime}$ be the set in $\Omega^{\prime}$ on which $\left[c X^{\prime}\right]_{*} \leqq 2 B^{1 / 2}$. Then $P^{\prime}\left(F^{\prime}\right) \geqq 3 / 4$ by Lemma 5. Also, $M>4 B$ implies $P^{\prime}\left(E^{\prime}\right) \geqq 3 / 4$. So $P^{\prime}\left(E^{\prime} \cap F^{\prime}\right) \geqq 1 / 2$. On replacing $E^{\prime}$ by $E^{\prime} \cap F^{\prime}$ in $(16)$ and $\left[c\left(X-X^{\prime}\right)\right]_{*}$ by $\left|[c X]_{*}-\left[c X^{\prime}\right]_{*}\right|$, we have

$$
\left(2 \mu_{q}\right)^{1 /(2 q)}(4 M)^{1 / 2} \geqq\left\{\int_{E} \int_{F^{\prime} \Omega E^{\prime}}[c X]_{*}-\left.\left[c X^{\prime}\right]_{*}\right|^{2 q} d \omega d \omega^{\prime}\right\}^{1 /(2 q)} .
$$

Minkowski's inequality now yields

$$
\begin{aligned}
\left(2 \mu_{q}\right)^{1 /(2 q)}(4 M)^{1 / 2} \geqq & \left\{\int_{E} \int_{E^{\prime} \cap F^{\prime}}[c X]_{*}^{2 q} d \omega d \omega^{\prime}\right\}^{1 /(2 q)} \\
& -\left\{\int_{E} \int_{E^{\prime} \cap}\left[c X^{\prime}\right]_{*}^{2 q} d \omega d \omega^{\prime}\right\}^{1 /(2 q)} \\
= & \left\{P^{\prime}\left(E^{\prime} \cap F^{\prime}\right) \int_{E}[c X]_{*}^{2 q} d \omega\right\}^{1 /(2 q)} \\
& -\left\{P(E) \int_{E^{\prime} \cap F^{\prime}}\left[c X^{\prime}\right]_{*}^{2 q} d \omega^{\prime}\right\}^{1 /(2 q)} \\
\geqq & \left\{P^{\prime}\left(E^{\prime} \cap F^{\prime}\right) \int_{E}[c X]_{*}^{2 q} d \omega\right\}^{1 /(2 q)}-2 B^{1 / 2}\left\{P^{\prime}\left(E^{\prime} \cap F^{\prime}\right)\right\}^{1 /(2 q)}
\end{aligned}
$$

The statement of the lemma follows easily, for $M>4 B$. 
Lemma 8. Suppose $p=2$. For every $\rho$ and every $\epsilon>0$ there are $a$ set $E$ and $a$ number $C$ with the property that

$$
\begin{gathered}
P(E)>1-\epsilon, \\
\int_{E} A_{P}\left\{[c X]_{*}\right\} d \omega<C
\end{gathered}
$$

for all $\left|c_{k}\right| \leqq 1$.

Choose $M$ so small that

$$
200 \rho M<1,
$$

then $N$ so large that

$$
\sum_{k=N+1}^{\infty} b_{k}<\frac{1}{2} \epsilon M
$$

The set $E_{1}$ on which

$$
\sum_{N+1}^{\infty}\left|X_{k}(\omega)\right|^{2} \leqq M
$$

has measure at least $1-\epsilon / 2$. Take $E$ to be the subset of $E_{1}$ on which

$$
\sum_{1}^{N}\left|X_{k}(\omega)\right|<L
$$

where $L$ is so large that (17) is true. We must now determine $C$ so that (18) is true. On $E$

$$
A_{\rho}\left\{[c X]_{*}\right\} \leqq A_{4 \rho}\{L\}+A_{4 \rho}\left\{\sup _{n>N}\left|\sum_{N+1}^{n} c_{k} X_{k}\right|\right\} .
$$

Thus it suffices to prove that

$$
\int_{E} A_{4 \rho}\left\{\sup _{n>N}\left|\sum_{N+1}^{n} c_{k} X_{k}\right|\right\} d \omega
$$

is bounded by a constant independent of the $c_{k}$. Now

$$
4 \sum_{q=1}^{\infty} \frac{\mu_{q}}{4^{q} q !}=2^{3 / 2} \pi^{-1 / 2} \int_{-\infty}^{\infty} e^{+u^{2} / 4} e^{-u^{2} / 2} d u-1=K<\infty,
$$

and (19-20), together with Lemma 7, yield

$$
\int_{E} A_{4 \rho}\left\{\sup _{n}\left|\sum_{N+1}^{n} c_{k} X_{k}\right|\right\} d \omega \leqq 4 \sum_{q=1}^{\infty} \frac{\mu_{q}(4 \rho)^{q}(9 M)^{q}}{q !} \leqq K .
$$


This proves the lemma, for $C$ may be taken to be $K+A_{4 p}\{L\}$.

Lemma $8^{\prime}$. Lemma 8 is true for $1 \leqq p<2$.

One defines $X_{n}^{\prime \prime \prime}(\omega)=X_{n}(\omega)$ if $\left|X_{n}(\omega)\right| \leqq 1$ and $X_{n}^{\prime \prime \prime}(\omega)=0$ otherwise; then $X_{n}^{\prime \prime}=X_{n}-X_{n}^{\prime \prime \prime}, M_{n}=\int X_{n}^{\prime \prime \prime} d \omega, X_{n}^{\prime}=X_{n}^{\prime \prime \prime}-M_{n}$, so that $X_{n}=X_{n}^{\prime}+X_{n}^{\prime \prime}$ $+M_{n}$. It turns out that the $X_{n}^{\prime}$ satisfy $\left(\mathrm{A}_{1-3}\right)$ with $p=2$, that for almost all $\omega$ the $X_{n}^{\prime \prime}$ are zero for sufficiently large $n$ (depending on $\omega$ ), and that $\sum\left|M_{n}\right|$ $\leqq B$. The proof is then completed as in Lemma 8 .

Lemma 1 , which is what we started out to prove, is hardly more than a rewording of Lemma $8^{\prime}$.

3. Proof of Theorems 1 and 2. In Lemma 1 set $c_{k}=e^{i \lambda_{k t}}$, multiply by $\left(1+t^{2}\right)^{-1}$, and integrate over $t$ :

$$
\int_{-\infty}^{\infty} \frac{d t}{1+t^{2}} \int_{\Omega} A_{\rho}\left\{Z_{*}(t, \omega)\right\} \psi(\omega) d \omega \leqq \pi .
$$

Hence (3) holds for almost all $\omega$, so that Theorem 1 is proved.

Inequality (3) shows incidentally that the approach of $Z_{n}$ to $Z$ is very "strong." Indeed, $\left|Z-Z_{n}\right| \leqq 2 Z_{*}$ and $Z_{n}(t, \omega) \rightarrow Z(t, \omega)$ for almost all $\omega$ and $t$; so

$$
\int_{-\infty}^{\infty} A_{\rho}\left\{Z(t, \omega)-Z_{n}(t, \omega)\right\}\left(1+t^{2}\right)^{-1} d t \rightarrow 0, \quad n \rightarrow \infty,
$$

for almost all $\omega$.

So far we have used no special properties of the exponential function. What we have said holds for any set of uniformly bounded functions. The situation is quite different concerning continuity. A good deal can be said when the $e^{i \lambda_{k} t}$ are replaced by functions whose moduli of continuity are known; the statements, however, are not nearly so precise as that of Theorem 2.

For the rest of this section we consider any $\omega$ for which (3) is true for all $\rho$, and drop $\omega$ from the notation.

Lemma 9. If $1<\lambda_{1}<\lambda_{2}<\cdots$ and $\lambda_{n} \rightarrow \infty$, then

$$
\sup _{-\infty<t<\infty} \frac{\left|Z_{n}(t)\right|}{1+t^{2}}=o\left(\lg \lambda_{n}\right)^{1 / 2}
$$

Let $a(r)=(d / d r) A_{\rho}(r)$ and let $b(s)=r$ be the inverse of $a(r)=s$. Then (Zygmund [11])

$$
\begin{aligned}
& B(s)=\int_{0}^{s} b(u) d u \leqq s \operatorname{Max}\left\{1, \rho^{-1 / 2}(\lg s)^{1 / 2}\right\}, \\
& x y \leqq A_{\rho}(x)+B(y), \quad x \geqq 0, y \geqq 0 \text {. }
\end{aligned}
$$


For $\lambda>0$, the function $e^{i \lambda w}(w+i)^{-2}$ is represented in the upper halfplane of $w=u+i v$ by its Poisson integral. So, for $v>0$,

$$
(w+i)^{-2} \sum_{1}^{n} X_{k} e^{i \lambda_{k} u-\lambda_{k} v}=\frac{1}{\pi} \int_{-\infty}^{\infty} Z_{n}\left(u^{\prime}\right) \frac{v}{\left(u-u^{\prime}\right)^{2}+v^{2}} \frac{d u}{\left(u^{\prime}+i\right)^{2}} .
$$

Noting that $v\left\{\left(u-u^{\prime}\right)^{2}+v^{2}\right\}^{-1} \leqq v^{-1}$ and keeping (21) and (22) in mind, we have: For $0<v<e^{-p}$,

$$
\begin{aligned}
\mid(w+ & i)^{-2} \sum_{1}^{n} X_{k} e^{i \lambda_{k} u-\lambda_{k} v} \mid \\
& \leqq \frac{1}{\pi} \int_{-\infty}^{\infty} A_{\rho}\left\{Z_{n}\left(u^{\prime}\right)\right\} \frac{d u^{\prime}}{\left|u^{\prime}+i\right|^{2}}+\frac{1}{\pi} \int_{-\infty}^{\infty} B\left\{\frac{v}{\left(u^{\prime}-u\right)^{2}+v^{2}}\right\} \frac{d u^{\prime}}{\left|u^{\prime}+i\right|^{2}} \\
& \leqq \pi^{-1} C_{\rho}+\pi^{-1} \rho^{-1 / 2}(\lg 1 / v)^{1 / 2} .
\end{aligned}
$$

Here $C_{\rho}$, furnished by Theorem 1 , is independent of $u, v$, and $n$. For $\epsilon>0$, first choose $\rho$ so that $\rho^{-1 / 2}<\epsilon$, then $v_{\rho}$ positive, $v_{\rho}<e^{-\rho}, C_{\rho}<\epsilon\left(\lg 1 / v_{\rho}\right)^{1 / 2}$. Thus for all $n$ and $u$

$$
\left|(w+i)^{-2} \sum_{1}^{n} X_{k} e^{i \lambda_{k} u-\lambda_{k} v}\right|<\epsilon(\lg 1 / v)^{1 / 2}, \quad 0<v<v_{\rho} .
$$

Fix $v$ for the moment. The function

$$
\left(u-i v^{\prime}-i v-i\right)^{-2} \sum_{1}^{n} X_{k} \exp \left\{-\lambda_{k} v-i\left(\lambda_{n}-\lambda_{k}\right) u-\left(\lambda_{n}-\lambda_{k}\right) v^{\prime}\right\}
$$

is analytic in $u-i v^{\prime}$ for $v^{\prime} \geqq 0$ and tends uniformly to zero as $|u|+\left|v^{\prime}\right| \rightarrow \infty$, $v^{\prime} \geqq 0$. Hence in $v^{\prime} \geqq 0$ it attains its maximum somewhere on the line $v^{\prime}=0$; when $v^{\prime}=v$ we are sure to get something smaller. So

$$
\begin{aligned}
\mid(u & -2 i v-i)^{-2} \sum_{1}^{n} X_{k} e^{i \lambda_{k} u} \mid \\
& =\left|(u-2 i v-i)^{-2} \exp \left(i \lambda_{n} u+\lambda_{n} v\right) \sum_{1}^{n} X_{k} \exp \left\{-\lambda_{k} v-i\left(\lambda_{n}-\lambda_{k}\right) u-\left(\lambda_{n}-\lambda_{k}\right) v\right\}\right| \\
& \leqq e^{\lambda_{n} v} \operatorname{supremum}_{v^{\prime} \geqq 0,-\infty<u<\infty} \mid\left(u-i v-i v^{\prime}-i\right)^{-2} \sum_{1}^{n} X_{k} \exp \left\{-\lambda_{k} v-i\left(\lambda_{n}-\lambda_{k}\right) u\right. \\
(23) & \left.\quad-\left(\lambda_{n}-\lambda_{k}\right) v^{\prime}\right\} \mid \\
& =e^{\lambda_{n} v} \sup _{-\infty<u<\infty}\left|(u-i v-i)^{-2} \sum_{1}^{n} X_{k} \exp \left(i \lambda_{k} u-\lambda_{k} v\right)\right| \\
& \leqq \epsilon e^{\lambda_{n} v}(\lg 1 / v)^{1 / 2} .
\end{aligned}
$$


If $n$ is large, $\lambda_{n}^{-1}<v_{\rho}$ and we may set $v=\lambda_{n}^{-1}$. Also $|u-2 i v-i|^{2}\left(1+u^{2}\right)^{-2}<2$ for small $v$. Thus (23) becomes for large $n$

$$
\left(1+u^{2}\right)^{-1}\left|\sum_{1}^{n} X_{k} e^{i \lambda_{k} u}\right| \leqq 5 \epsilon\left(\lg \lambda_{n}\right)^{1 / 2} .
$$

This is the statement of the lemma.

Theorem 2 follows at once. Let $\beta=(1+\epsilon) / 2$. Hypothesis (6) implies that the random variables $\left(\lg \lambda_{n}\right)^{\beta} X_{n}$ satisfy $\left(\mathrm{A}_{1-3}\right)$, so that according to Lemma 9

$$
\tilde{Z}_{n}(t)=\sum_{1}^{n} X_{k}\left(\lg \lambda_{k}\right)^{\beta} e^{i \lambda_{k} t}=o\left\{\left(1+t^{2}\right)\left(\lg \lambda_{n}\right)^{1 / 2}\right\} .
$$

The theorem is obtained by writing

$$
\begin{aligned}
Z_{n}-Z_{m}= & \sum_{k=m}^{n-1}\left\{\left(\lg \lambda_{k}\right)^{-\beta}-\left(\lg \lambda_{k+1}\right)^{-\beta}\right\} \tilde{Z}_{k} \\
& -\left(\lg \lambda_{m}\right)^{-\beta} \tilde{Z}_{m-1}+\left(\lg \lambda_{n}\right)^{-\beta} \tilde{Z}_{n} .
\end{aligned}
$$

4. Continuity of random integrals. We consider a family $X(\lambda, \omega)$ of random variables and define the random function $X(I, \omega)$ of intervals by

$$
X(I, \omega)=X\left(\lambda_{2}, \omega\right)-X\left(\lambda_{1}, \omega\right), \quad I: \lambda_{1}<\lambda \leqq \lambda_{2} .
$$

The place of the $b_{n}$ of $\S 1$ is taken by

$$
B(I)=\int_{\Omega}|X(I, \omega)|^{2} d \omega .
$$

Four restrictions are imposed on $X(I)$ :

( $\left.\mathrm{B}_{1}\right) X(I, \omega), \cdots, X\left(I_{n}, \omega\right)$ are independent if $I_{j} \cap I_{k}=0$ when $j \neq k$.

$\left(\mathrm{B}_{2}\right) \int_{\Omega} X(I, \omega) d \omega=0$ for every $I$.

$\left(\mathrm{B}_{3}\right) B(I)$ is bounded.

$\left(\mathrm{B}_{4}\right) X(\lambda, \omega)$ is measurable on $(\lambda) \times \Omega$. For each $\omega$ the function $X(\lambda, \omega)$ has discontinuities only of the first kind (as function of $\lambda$ ). For every $\omega$ and every $\lambda, X(\omega, \lambda+h) \rightarrow X(\omega, \lambda)$ as $h$ decreases to 0 .

Doob [3] has shown that if $\tilde{X}$ satisfies $\left(B_{1-3}\right)$ there is an $X$ which agrees with $\tilde{X}$ up to an $\Omega$ set of measure zero for each $I$ and almost satisfies $\left(B_{1-4}\right)$. There may be a fixed enumerable set $\lambda_{1}, \lambda_{2}, \cdots$ at which $X(\lambda, \omega)$ is not continuous on the right; the removal of these discontinuities requires only a trivial modification of $X$, which we suppose performed. The matter is unimportant, since we introduce $\left(B_{4}\right)$ only to define integrals in an elementary way.

For almost all $\omega$, the function $X(\lambda, \omega)$ is bounded, has only an enumerable number of discontinuities, and tends to a limit as $\lambda \rightarrow \pm \infty$. Thus $X(\lambda, \omega)$ is Riemann integrable, so that 


$$
\int_{\Delta_{1}}^{\Delta_{2}} X(\lambda, \omega) e^{i \lambda t} d \lambda
$$

exists as a limit of Riemann sums and represents an integral function of $t$. We define

$$
Z\left(t, \omega, \Lambda_{1}, \Lambda_{2}\right)=\int_{\Lambda_{1}}^{\Lambda_{2}} e^{i \lambda t} X(d \lambda, \omega)
$$

to be the limit of Riemann-Stieltjes sums. That (25) is defined, that it is an integral function of $t$, and that

$$
Z\left(t, \omega, \Lambda_{1}, \Lambda_{2}\right)=X\left(\Lambda_{2}, \omega\right) e^{i \Lambda_{2} t}-X\left(\Lambda_{1}, \omega\right) e^{i \Lambda_{1} t}-i t \int_{\Lambda_{1}}^{\Lambda_{2}} X(\lambda, \omega) e^{i \lambda t} d \lambda
$$

are all consequences of the existence of $(24)$. Later on $(\$ \S 5,8,10)$ we shall often integrate or differentiate under the integral sign; these operations are easily justified if one recalls (26).

We use a notation corresponding to that for series:

$$
\begin{gathered}
Z_{*}(t, \omega)=\sup _{\Lambda_{1}<\Lambda_{2}}\left|\int_{\Lambda_{1}}^{\Delta_{2}} e^{i \lambda t} X(d \lambda, \omega)\right| . \\
Z(t, \omega)=\lim _{\Lambda_{\rightarrow \infty}} \int_{-\Lambda}^{\Delta} e^{i \lambda t} X(d \lambda, \omega) .
\end{gathered}
$$

THEOREM 3. If for some $\epsilon>0$

$$
\int_{-\infty}^{\infty}\left\{\lg \left(1+\lambda^{2}\right)\right\}^{1+\epsilon} B(d \lambda)<\infty,
$$

then for almost all $\omega$,

$$
\frac{Z\left(t, \omega, \Lambda_{1}, \Lambda_{2}\right)}{1+t^{2}} \rightarrow \frac{Z(t, \omega)}{1+t^{2}}
$$

uniformly in $t$ as $\Lambda_{1} \rightarrow-\infty, \Lambda_{2} \rightarrow \infty$. In particular, $Z(t, \omega)$ is continuous in $t$ for almost all $\omega$.

Let $Z(t, \omega, \Lambda)=Z(t, \omega, 1, \Lambda)$. Clearly it suffices to prove that $\left(1+t^{2}\right)^{-1} Z(t, \omega, \Lambda)$ tends uniformly in $t$ to a limit as $\Lambda \rightarrow \infty$.

Let $\sum \epsilon_{m}=\epsilon<1$. Define

$$
Y_{n}(\lambda, \omega)=X\left(\frac{k}{n}, \omega\right), \quad \frac{k}{n} \leqq \lambda<\frac{k+1}{n} .
$$

For each $\omega$ and each integer $m$, 


$$
\int_{m}^{m+1}\left|Y_{n}(\lambda, \omega)-X(\dot{\lambda}, \omega)\right| d \lambda \rightarrow 0, \quad n \rightarrow \infty .
$$

Hence there are integers $n_{m}$ and sets $E_{m} \subset \Omega$ such that $P\left(E_{m}\right)>1-\epsilon_{m}$ and

$$
\int_{m}^{m+1}\left|Y_{n_{m}}(\lambda, \omega)-X(\lambda, \omega)\right| d \lambda<\epsilon_{m}, \quad \omega \in E_{m} .
$$

Let $Y(\lambda, \omega)=Y_{n_{m}}(\lambda, \omega)$ for $m \leqq \lambda<m+1$ and let $E=\cap E_{m}$. Clearly $P(E)>1-\epsilon$ and, for $\omega \in E$,

$$
\begin{gathered}
\int_{1}^{\infty}|Y(\lambda, \omega)-X(\lambda, \omega)| d \lambda<\epsilon, \\
\int_{\Lambda_{1}}^{\Delta_{2}} e^{i \lambda t} X(d \lambda)-\int_{\Lambda_{1}}^{\Lambda_{2}} e^{i \lambda t} Y(d \lambda)=\left\{X\left(\Lambda_{2}\right)-Y\left(\Lambda_{2}\right)\right\} e^{i \Lambda_{2} t} \\
-\left\{X\left(\Lambda_{1}\right)-Y\left(\Lambda_{1}\right)\right\} e^{i \Lambda_{1} t}+i t \int_{\Lambda_{1}}^{\Delta_{2}}\{Y(\lambda)-X(\lambda)\} e^{i \lambda t} d \lambda \\
=P+Q+t R .
\end{gathered}
$$

Here $P, Q, R$ are uniformly bounded in $\Lambda_{1}, \Lambda_{2}, t$ and tend to zero uniformly in $t$ as $\Lambda_{1}$ and $\Lambda_{2}$ tend to $\infty$, provided only that $\omega \in E$. Also, it is easy to see that

$$
\int_{1}^{\Lambda} e^{i \lambda t} Y(d \lambda, \omega)
$$

can be written as a series $\sum Y_{k}(\omega) e^{i \lambda_{k} t}$ with the $Y_{k}$ satisfying the hypotheses of Theorem 2. Thus

$$
\left(1+t^{2}\right)^{-1} \int_{1}^{\Delta} e^{i \lambda t} Y(d \lambda)
$$

tends uniformly in $t$ to its limit for almost all $\omega \in \Omega$. All in all, for almost every $\omega$ in $E$

$$
\left(1+t^{2}\right)^{-1} \int_{\Lambda_{1}}^{\Lambda_{2}} e^{i \lambda t} X(d \lambda, \omega)=o(1), \quad \Lambda_{1} \rightarrow \infty, \Lambda_{2} \rightarrow \infty,
$$

uniformly in $t$. As the measure of $E$ can be taken arbitrarily close to 1 , we conclude that the theorem is true.

If we had considered the $L^{2}$ space over $(t) \times \Omega$ (measure $\left(1+t^{2}\right)^{-1} d t d \omega$ ) and defined (25) as a strong limit of Riemann-Stieltjes sums, we should not have needed $\left(B_{4}\right)$. The integral, however, would have been defined only "almost everywhere" and we should have to pick a suitable function out of this equivalence class in order to make statements like those in the theorem. 
5. The modulus of continuity. The hypotheses of several later theorems can be expressed either in terms of $B(I)$ or in terms of the autocorrelation function

$$
\phi(t)=\int_{\Omega} \bar{Z}(s, \omega) \cdot Z(t+s, \omega) d \omega=\int_{-\infty}^{\infty} e^{i t \lambda} B(d \lambda)
$$

First we recall that the existence of the moment of order $2 q$ of $B$ is equivalent to the existence of the derivative of order $2 q$ of $\phi$ when $q$ is a positive integer. Next

$$
\begin{aligned}
& \beta \int_{0}^{\infty} \frac{2 \phi(0)-\phi(t)-\phi(-t)}{t^{1+\beta}} d t \\
&=2 \Gamma(1-\beta) \sin (1-\beta) \frac{\pi}{2} \int_{-\infty}^{\infty}|\lambda| \beta B(d \lambda)
\end{aligned}
$$

if $0<\beta<2$-the assertion being that if one member is finite the other is finite also and has the same value. We may assume that $B(I)=B(-I)$ (else replace $B(I)$ by $B(I)+B(-I))$. Then

$$
\phi(t)=2 \int_{0}^{\infty} \cos t \lambda B(d \lambda) .
$$

Now, a familiar formula reads

$$
\int_{0}^{\infty} \frac{1-\cos \lambda t}{t^{1+\beta}} d t=\beta^{-1} \Gamma(1-\beta) \sin (1-\beta) \frac{\pi}{2} \cdot|\lambda|^{\beta}, \quad 0<\beta<2 .
$$

Since everything is positive, we run into no trouble if we multiply by $B(d \lambda)$ and integrate. The result is

$$
\begin{aligned}
\int_{0}^{\infty} B(d \lambda) \int_{0}^{\infty} \frac{1-\cos \lambda t}{t^{1+\beta}} d t & =\int_{0}^{\infty} \frac{d t}{t^{1+\beta}} \int_{0}^{\infty}(1-\cos \lambda t) B(d \lambda) \\
& =\frac{1}{2} \int_{0}^{\infty} \frac{\phi(0)-\phi(t)}{t^{1+\beta}} d t \\
& =\beta^{-1} \Gamma(1-\beta) \sin (1-\beta) \frac{\pi}{2} \int_{0}^{\infty} \lambda^{\beta} B(d \lambda) .
\end{aligned}
$$

The symmetry of $B(I)$ and (33) give (32). Incidentally (32) and differentiation permit any absolute moment of positive order of $B(I)$ to be expressed in terms of its characteristic function.

We say that $f(t)$ belongs to $H_{\alpha}$ if the following is true: Given any finite interval $(a, b)$ there are two constants $C$ and $D$ such that 


$$
|f(t+h)-f(t)| \leqq C h^{\alpha}\left(\lg \frac{D}{h}\right)^{1 / 2}, \quad a<t<t+h<b .
$$

If all functions of a set satisfy (34) with the same $C$ and $D$, we say they belong uniformly to $H_{\alpha}$.

Theorem 4. Suppose that $0<\alpha \leqq 1$ and that

$$
\int_{-\infty}^{\infty}|\lambda|^{2 \alpha} B(d \lambda)<\infty .
$$

Then for almost all $\omega$ the $Z\left(t, \omega, \Lambda_{1}, \Lambda_{2}\right)$ and $Z(t, \omega)$ belong uniformly to $H_{\alpha}$.

It suffices to prove that the functions $Z(t, \omega, \Lambda)=Z(t, \omega, 1, \Lambda)$ belong uniformly to $H_{\alpha}$. In this case we can formulate the statement in a way which makes the details of the proof a little easier: Suppose that $X(I, \omega)$ satisfies $\left(\mathrm{B}_{1-4}\right)$-but not necessarily (35)-and define

$$
Z^{\alpha}(t, \omega, \Lambda)=\int_{1}^{\Lambda} \frac{e^{i \lambda t}}{(i \lambda)^{\alpha}} X(d \lambda, \omega), \quad 1<\Lambda<\infty ;
$$

then the $Z^{\alpha}(t, \omega, \Lambda)$ belong uniformly in $\Lambda$ to $H_{\alpha}$. It is this second form which we shall prove.

Let us first settle the case $\alpha=1$. Consider any $\omega$ for which

$$
\int_{-T}^{T} A_{\rho}\left\{Z_{*}(t, \omega)\right\} d t=K(\omega, \rho, T)=K<\infty
$$

for every $T, \rho$ and drop $\omega$ from the notation. (That (36) holds for almost all $\omega$ follows from Theorem 1 and the approximation used in $\$ 4$. Indeed, one can prove a statement for integrals similar to Lemma 1.) Now

$$
\frac{1}{h}\left\{Z^{1}(t+h, \Lambda)-Z^{1}(t, \Lambda)\right\}=\frac{1}{h} \int_{t}^{t+h} Z(u, \Lambda) d u .
$$

Since $A_{\rho}(u)$ is convex, (37) and Jensen's inequality imply that

$$
\begin{aligned}
A_{\rho}\left\{\frac{Z^{1}(t+h)-Z^{1}(t)}{h}\right\} & =A_{\rho}\left\{\frac{1}{h} \int_{t}^{t+h} Z(u) d u\right\} \\
& \leqq \frac{1}{h} \int_{t}^{t+h} A_{\rho}\left\{Z_{*}(u)\right\} d u \\
& \leqq K / h, \quad-T<t<t+h<T .
\end{aligned}
$$

A slight rearrangement yields

$$
\left|Z^{1}(t+h, \Lambda)-Z^{1}(t, \Lambda)\right| \leqq \rho^{-1 / 2} h\left\{\lg \left(\frac{K}{h}+1\right)\right\}^{1 / 2} .
$$


This proves (34) when $\alpha=1$ and incidentally shows that for small $h$ the $C$ and $D$ can be chosen small.

When $0<\alpha<1$ we express $Z^{\alpha}$ as the sum of a well behaved function and of a fractional derivative of $Z^{1}(t, \Lambda)$. From $\int_{0}^{\infty} e^{i t} t^{\alpha-1} d t=i^{\alpha} \Gamma(\alpha)$ we see that

$$
\frac{1}{\Gamma(\alpha)} \int_{0}^{1} u^{\alpha-1} e^{i \lambda(t-u)} d u=\frac{e^{i \lambda t}}{(i \lambda)^{\alpha}}+F(\lambda) \frac{e^{i \lambda t}}{\lambda}
$$

where $F(\lambda)$ is bounded and continuous. Thus

$$
\frac{1}{\Gamma(\alpha)} \int_{0}^{1} u^{\alpha-1} Z(t-u, \Lambda) d u=\int_{1}^{\Delta} \frac{e^{i \lambda t}}{(i \lambda)^{\alpha}} X(d \lambda)+\int_{1}^{\Delta} \frac{e^{i \lambda t}}{i \lambda} F(\lambda) X(d \lambda) .
$$

Since

$$
\int_{1}^{\infty}|F(\lambda)|^{2} B(d \lambda)<\infty
$$

we conclude from what has already been proved for $\alpha=1$ that the second term on the right in (39) belongs to $H_{1}$ uniformly in $\Lambda$. So we need consider only the term on the left.

The function $Z^{1}(t)-Z^{1}(t-u)$ is an indefinite integral of $Z(t-u)$ (as function of $u$ ) and vanishes at $u=0$ like $u(\lg 1 / u)^{1 / 2}$. Hence, integrating by parts, we have

$$
\int_{0}^{1} u^{\alpha-1} Z(t-u) d u=Z^{1}(t)-Z^{1}(t-1)+(1-\alpha) \int_{0}^{1}\left\{Z^{1}(t)-Z^{1}(t-u)\right\} \frac{d u}{u^{2-\alpha}} .
$$

The first two terms on the right again belong to $H_{1}$ uniformly in $\Lambda$. Writing

$$
Y(t, \Lambda)=\int_{0}^{1}\left\{Z^{1}(t, \Lambda)-Z^{1}(t-u, \Lambda)\right\} \frac{d u}{u^{2-\alpha}},
$$

we have

$$
Y(t+h)-Y(t)=\int_{0}^{1}\left\{Z^{1}(t+h)-Z^{1}(t+h-u)-Z^{1}(t)+Z^{1}(t-u)\right\} \frac{d u}{u^{2-\alpha}} .
$$

We must show this to be less than $C h^{\alpha}(\lg D / h)^{1 / 2}$ for, say, $-T<t<t+h<T$. If $0<h<1$, then

$$
\begin{aligned}
& \int_{0}^{h}\left\{\left|Z^{1}(t+h)-Z^{1}(t+h-u)\right|+\left|Z^{1}(t)-Z^{1}(t-u)\right|\right\} u^{\alpha-2} d u \\
& \leqq \int_{0}^{1} C_{1} u\left(\lg C_{2} / u\right)^{1 / 2} u^{\alpha-2} d u \leqq C_{3} h^{\alpha}\left(\lg C_{4} / h\right)^{1 / 2}
\end{aligned}
$$




$$
\begin{aligned}
& \int_{h}^{1}\left\{\left|Z^{1}(t+h)-Z^{1}(t)\right|+\left|Z^{1}(t+h-u)-Z^{1}(t-u)\right|\right\} u^{\alpha-2} d u \\
& \leqq \int_{h}^{1} C_{1} h\left(\lg C_{2} / h\right)^{1 / 2} u^{\alpha-2} d u \leqq C_{5} h^{\alpha}\left(\lg C_{2} / h\right)^{1 / 2}
\end{aligned}
$$

Here the constants $C_{1}$ and $C_{2}$, which are furnished by what has been proved for $\alpha=1$, are independent of $\Lambda$. If $h>1$ we have a similar estimate without the need of breaking up the range of integration. Thus $Y(t, \Lambda)$ belongs to $H_{\alpha}$ uniformly in $\Lambda$. Tracing the steps backwards we see that $Z^{\alpha}(t, \Lambda)$ also belongs to $H_{\alpha}$ uniformly in $\Lambda$.

6. Another inequality. Our study of $Z(t, \omega)$ for large $t$ is based on the following inequality.

Lemma 10. Suppose that $X_{1}, X_{2}, \cdots$ satisfy $\left(\mathrm{A}_{1-3}\right)$. Then for almost all $\omega$ and for all $\rho$

$$
\sup _{T} \frac{1}{T} \int_{0}^{T} A_{\rho}\left\{Z_{*}(t, \omega)\right\} d t<\infty
$$

If also $p=2$ in $\left(\mathrm{A}_{3}\right)$ and $1000 \rho B<1$, and if $G_{\rho, a}$ is the set on which the left member of (40) is less than $a$, then

$$
P\left\{G_{\rho, a}\right\} \geqq 1-\left(2+300 a^{-1}\right)(\rho B)^{1 / 3} \text {. }
$$

We shall prove (41) first. Then (40) and a somewhat weaker inequality for integrals follow without difficulty. The principle steps are given as lemmas.

LEMMA 11. Let $S$ be a space of total measure $1, s \rightarrow s^{t}$ a measure preserving flow on $S, f(s) \geqq 0$, and $T(s) \neq 0$. Then

$$
\int_{S} \frac{d s}{T(s)} \int_{0}^{T(s)} f\left(s^{t}\right) d t \leqq 2\left\{\int_{S} f^{2}(s) d s\right\}^{1 / 2} .
$$

This is a corollary of the maximal ergodic theorem.

LEMMA 12. Let $\phi_{k}(u)$ be the Rademacher functions, $\lambda_{k}$ real numbers, $T(u)$ $\neq 0, \sum\left|a_{k}\right|^{2}=D$, and $20 D \rho<1$. Then

$$
\int_{0}^{1} \frac{d u}{T(u)} \int_{0}^{T(u)} A_{\rho}\left\{\left[a \phi(u) e^{i \lambda t}\right]_{*}\right\} d t \leqq 20(\rho D)^{1 / 2} .
$$

The proof is rather long. In Lemma 11 take $S=S_{1} \times S_{2} \times \cdots$, each $S_{k}$ being the interval $-1 / 2<s_{k} \leqq 1 / 2$ provided with ordinary Lebesgue measure. The flow $s \rightarrow s^{t}$ defined by

$$
s_{k}^{t} \equiv s_{k}+(2 \pi)^{-1} \lambda_{k} t(\bmod 1), \quad 1 / 2<s_{k}^{t} \leqq 1 / 2,
$$

clearly preserves measure. The functions $e^{2 \pi i s_{k}}$ (considered as functions on $S$ ) 
are a set of random variables satisfying the hypotheses of Lemma 4; hence

$$
\begin{aligned}
\int_{S} A_{\rho}\left\{\left[a e^{2 \pi i s}\right]_{*}\right\} d s & \leqq\left(\frac{2}{\pi}\right)^{1 / 2} \int_{-\infty}^{\infty}\left(e^{\rho D u^{2}}-1\right) e^{-u^{2} / 2} d u \\
& \leqq 4 \rho D
\end{aligned}
$$

if $4 \rho D<1$. Thus in Lemma 11 we may set $f(s)=A_{\rho}\left\{\left[a e^{2 \pi i s}\right]_{*}\right\}$ to obtain

$$
\begin{aligned}
& \int_{S} \frac{d s}{T(s)} \int_{0}^{T(s)} A_{\rho}\left\{\left[a e^{2 \pi i s} e^{i \lambda t}\right]_{*}\right\} d t \\
& \leqq 4\left\{\int_{S} A_{\rho}^{2}\left\{\left[a e^{2 \pi i s}\right]_{*}\right\} d s\right\}^{1 / 2} \\
&=4\left\{\int_{S} A_{2 \rho}\left\{\left[a \dot{e}^{2 \pi i s}\right]_{*}\right\} d s\right\}^{1 / 2} \\
& \leqq 12(\rho D)^{1 / 2}
\end{aligned}
$$$$
8 \rho D<1 .
$$

Let now $U_{k}$ be the space consisting of the two elements +1 and -1 , to each of which is attributed measure $1 / 2$, and let $U=U_{1} \times U_{2} \times \ldots$ The transformation $s \rightarrow(\bar{s}, u)$ defined by $\left(\bar{s}_{k}, u_{k}\right)=\left(2 s_{k},+1\right)$ if $-1 / 4<s_{k} \leqq 1 / 4$ and by $\left(\bar{s}_{k}, u_{k}\right)=\left(2 s_{k}-\operatorname{sgn} s_{k},-1\right)$ otherwise maps $S$ on $S \times U$ with preservation of measure and carries $e^{2 \pi i s k}$ into $u_{k} e^{\pi i s k}$. Under this transformation (45) becomes

$$
\int_{U} d u \int_{S} \frac{d s}{T(s, u)} \int_{0}^{T(s, u)} A_{\rho}\left\{\left[a u e^{\pi i s} e^{i \lambda t}\right]_{*}\right\} d t \leqq 12(\rho D)^{1 / 2}, \quad 8 \rho D<1 .
$$

Now, $A_{\rho}\{x\}$ is an increasing convex function of $|x|$ and

$$
\int_{S}\left[a u e^{i \lambda t} e^{\pi i s}\right]_{*} d s \geqq\left[a u e^{i \lambda t} \int_{S} e^{\pi i s} d s\right]_{*}=\frac{2}{\pi}\left[a u e^{i \lambda t}\right]_{*}
$$

Keeping these facts in mind, we apply Jensen's inequality to (46), first specializing $T(s, u)$ to $T(u)$ :

$$
\begin{aligned}
12(\rho D)^{1 / 2} & \geqq \int_{U} \frac{d u}{T(u)} \int_{0}^{T(u)} d t \int_{S} d s A_{\rho}\left\{\left[a u e^{\pi i s} e^{i \lambda t}\right]_{*}\right\} \\
& \geqq \int_{U} \frac{d u}{T(u)} \int_{0}^{T(u)} A_{\rho}\left\{\int_{S}\left[a u e^{\pi i s} e^{i \lambda t}\right]_{*} d s\right\} d t \\
& \geqq \int_{U} \frac{d u}{T(u)} \int_{0}^{T(u)} A_{\rho}\left\{\frac{2}{\pi}\left[a u e^{i \lambda t}\right]_{*}\right\} d t, \quad 8 \rho D<1 .
\end{aligned}
$$

Since $2^{2} / \pi^{2}>4 / 10$, and since there is a measure preserving transformation of $U$ on $\langle 0,1\rangle$ which carries $u_{k}$ into $\phi_{k},(47)$ implies the lemma. 
Lemma 13. Suppose that $X_{1}, X_{2}, \cdots$ are symmetric and satisfy $\left(\mathrm{A}_{1-3}\right)$ with $p=2$, that $T(\omega) \neq 0$, and that $D>0$. Let $E$ be the set on which $\sum\left|X_{k}\right|^{2} \leqq D$. Then

$$
\int_{E} \frac{d \omega}{T(\omega)} \int_{0}^{T(\omega)} A_{\rho}\left\{Z_{*}(t, \omega)\right\} d t \leqq 20(\rho D)^{1 / 2}, \quad 20 \rho D<1 .
$$

The proof is like that of Lemma 6.

Lemma 14. Suppose that $X_{1}, X_{2}, \cdots$ satisfy $\left(\mathrm{A}_{1-3}\right)$ with $p=2$, that $D>4 B$, that $T(\omega) \neq 0$, and that $160 D \rho<1$. Define $E$ as in Lemma 13. Then

$$
\int_{E} \frac{d \omega}{T(\omega)} \int_{0}^{T(\omega)} A_{\rho}\left\{Z_{*}(t, \omega)\right\} d t<400(\rho D)^{1 / 2}
$$

We take replicas $\Omega^{\prime}, X_{k}^{\prime}\left(\omega^{\prime}\right), E^{\prime}$ of $\Omega, X_{k}(\omega), E$. Since $\sum\left|X_{k}-X_{k}^{\prime}\right|^{2}<4 D$ on $E \times E^{\prime}$,

$$
\int_{E} \frac{d \omega}{T(\omega)} \int_{E^{\prime}} d \omega^{\prime} \int_{0}^{T(\omega)} A_{2 \rho}\left\{\left[\left(X-X^{\prime}\right) e^{i \lambda t}\right]_{*}\right\} d t<60(\rho D)^{1 / 2}
$$

Let $F_{t}^{\prime}$ be the set in $\Omega^{\prime}$ on which $\left[X^{\prime}\left(\omega^{\prime}\right) e^{i \lambda_{k} t}\right]_{*}<2 B^{1 / 2}$. Then $P^{\prime}\left(F_{t}^{\prime}\right) \geqq 3 / 4$; and also $P^{\prime}\left(F_{t}^{\prime} \cap E^{\prime}\right) \geqq 1 / 2$, for $P^{\prime}\left(E^{\prime}\right)=P(E) \geqq 3 / 4$. If $\omega^{\prime} \in F_{\imath}^{\prime}$

$$
\left[\left(X(\omega)-X^{\prime}\left(\omega^{\prime}\right)\right) e^{i \lambda t}\right]_{*}^{2} \geqq \frac{1}{2}\left[X(\omega) e^{i \lambda t}\right]_{*}^{2}-4 B,
$$

so that

$$
\begin{aligned}
A_{2 \rho}\left\{\left[\left(X(\omega)-X^{\prime}\left(\omega^{\prime}\right)\right) e^{i \lambda t}\right]_{*}\right\} & \geqq \exp \left\{\rho\left(\left[X(\omega) e^{i \lambda t}\right]_{*}^{2}-8 B\right)\right\}-1 \\
& \geqq e^{-8 \rho B} A_{\rho}\left\{\left[X e^{i \lambda t}\right]_{*}\right\}-24 \rho B e^{-8 \rho B} .
\end{aligned}
$$

(Note that $8 \rho B<1$ and $e^{x}<1+3 x$ for $0<x<1$.) This being so, replace $E^{\prime}$ by $E^{\prime} \cap F_{t}^{\prime}$ in (49):

$$
\begin{aligned}
60(\rho D)^{1 / 2} & >\int_{E} \frac{d \omega}{T(\omega)} \int_{0}^{T(\omega)} d t \int_{E^{\prime} \cap F_{t}^{\prime}} d \omega^{\prime} A_{2 \rho}\left\{\left[\left(X(\omega)-X\left(\omega^{\prime}\right)\right) e^{i \lambda t}\right]_{*}\right\} \\
& \geqq e^{-8 \rho B} \int_{E} \frac{d \omega}{T(\omega)} \int_{0}^{T(\omega)} d t A_{\rho}\left\{\left[X(\omega) e^{i \lambda t}\right]_{*}\right\} \int_{E^{\prime} \cap_{F_{t}^{\prime}}} d \omega^{\prime}-24 \rho B e^{-8 \rho B} \\
& \geqq \frac{1}{2} e^{-8 \rho B} \int_{E} \frac{d \omega}{T(\omega)} \int_{0}^{T(\omega)} A_{\rho}\left\{\left[X(\omega) e^{i \lambda t}\right]_{*}\right\} d t-24 B e^{-8 \rho B} .
\end{aligned}
$$

Inequality (48) is nothing but a restatement of (50), account being taken that $D>4 B, \rho D<(\rho D)^{1 / 2}, e^{8 \rho B}<3$.

It follows from (48) that 


$$
\begin{aligned}
P\left\{\frac{1}{T(\omega)} \int_{0}^{T(\omega)} A_{\rho}\left\{Z_{*}(t, \omega)\right\} d t<a\right\} & \geqq P(E)-400(\rho D)^{1 / 2} a^{-1} \\
& \geqq 1-B D^{-1}-400(\rho D)^{1 / 2} a^{-1}
\end{aligned}
$$

Since $T(\omega)$ may be chosen at will the left member may be replaced by $P\left\{G_{\rho, a}\right\}$, where $G_{\rho, a}$ is the set defined in Lemma 10 . When $1000 \rho B<1$ we may set $D=2^{-1} B^{2 / 3} \rho^{-1 / 3}$ and still satisfy the condition $D>4 B$ and $160 D \rho<1$ of Lemma 14. Then (51) becomes (41); so the last part of Lemma 10 is proved. In order to prove the first part one proceeds just as in the proof of Lemmas 8 and $8^{\prime}$. There is so little change that we omit the argument.

Lemma 15. If $X(\lambda, \omega)$ satisfies $\left(B_{1-4}\right)$ and $1000 \rho B<1$, then (41) is true. In particular, for almost every $\omega$ and for some $\rho=\rho(\omega)>0$ the inequality (40) is true.

We shall see later that when $Z(t, \omega)$ is an integral, (40) may not be true for all $\rho>0$.

In order to prove (41) for integrals it suffices to prove

$$
\begin{aligned}
P\left\{\frac{1}{T(\omega)} \int_{0}^{T(\omega)} A_{\rho}\left\{\int_{0}^{\Delta(\omega, t)} e^{i \lambda t} X(d \lambda, \omega)\right\} d t\right. & <a\} \\
& \geqq 1-\left(2+300 a^{-1}\right)(\rho B)^{1 / 3}
\end{aligned}
$$

for arbitrary $T(\omega)$ and $\Lambda(\omega, t)$. The method of approximation by series used in \$4, together with (41) and Fatou's theorem, yields (52) without trouble, though the details are rather tedious. It is at this point that it becomes important to have a supremum and not a limit in (41).

7. The mean behavior of $Z(t, \omega)$. Let $\mathcal{X}_{\rho}$ be the class of functions $f(t)$ for which

$$
\sup _{T \neq 0} \frac{1}{T} \int_{0}^{T} A_{\rho}\{f(t)\} d t<\infty .
$$

In $\mathcal{X}_{\rho}$ define a "distance" $(f, g)_{p}$ by

$$
(f, g)_{\rho}=\left\{\frac{1}{\rho} \lg \sup _{T} \frac{1}{T} \int_{0}^{T} \exp \left\{\rho|f-g|^{2}\right\} d t\right\}^{1 / 2} .
$$

The triangle inequality is not satisfied, but for any elements $f, g, h$ of $\mathcal{X}_{\rho}$

$$
(f, g)_{\rho} \leqq(f, h)_{4 \rho}+(h, g)_{4 \rho} .
$$

The article [5] by Jessen may be consulted for further information about $\mathcal{X}_{\rho}$.

TheOREM 5. If $X_{1}, X_{2}, \cdots$ satisfy $\left(\mathrm{A}_{1-3}\right)$, then for almost all $\omega$ the functions $Z_{n}(t, \omega)$ and $Z(t, \omega)$ belong to $\mathcal{X}_{\rho}$ and $\left(Z_{n}(\omega, t), Z(t, \omega)\right)_{\rho} \rightarrow 0$ for every $\rho$. In par- 
ticular $Z(t, \omega)$ is (for almost all $\omega$ ) an almost periodic function belonging to every $B^{p}$.

The second statement is a corollary to the first, for convergence in $\mathcal{X}_{\boldsymbol{p}}$ implies convergence in $B^{p}$, and $Z_{n}(t, \omega)$ is a trigonometric polynomial. In order to prove the first statement, note that for $n>N$

$$
\left|Z(t, \omega)-Z_{n}(t, \omega)\right| \leqq 2 \sup _{l \geqq N}\left|\sum_{N}^{l} X_{k}(\omega) e^{i \lambda_{k} t}\right| .
$$

Setting

$$
B_{N}=\sum_{n=N}^{\infty} b_{n}
$$

we have, according to Lemma 10 ,

$$
\begin{aligned}
P\left\{\sup _{T} \frac{1}{T} \int_{0}^{T} A_{4 \rho}\left\{\sup _{l \geqq N}\left|\sum_{N}^{l} X_{k}(\omega) e^{i \lambda_{k} t}\right|\right\} d t \leqq \epsilon\right\} & \\
& \geqq 1-\left(2+300 \epsilon^{-1}\right)\left(4 \rho B_{N}\right)^{1 / 3}
\end{aligned}
$$

which is close to 1 for large $N$. Thus

$$
P\left\{\sup \frac{1}{T} \int_{0}^{T} A_{\rho}\left\{Z(t, \omega)-Z_{n}(t, \omega)\right\} d t \leqq \epsilon\right\} \geqq 1-\epsilon
$$

for large $N$ and all $n>N$.

The proof of the following theorem is similar.

Theorem 6. If $X(\lambda, \omega)$ satisfies $\left(\mathrm{B}_{1-4}\right)$, then for almost all $\omega$ there is a $\rho=\rho(\omega)>0$ such that $\left(Z(t, \omega), Z\left(t, \omega, \Lambda_{1}, \Lambda_{2}\right)\right)_{\rho} \rightarrow 0$ as $\Lambda_{1} \rightarrow-\infty, \Lambda_{2} \rightarrow \infty$. In particular, if $X(\lambda, \omega)$ (for almost every $\omega$ ) changes only by jumps and has only a finite number of jumps in every finite interval, then $Z(t, \omega)$ is almost periodic.

It would be interesting to find out whether $Z(t, \omega)$ has a spectrum, in the sense of Wiener, for almost every $\omega$. It would then follow that the limit

$$
\lim _{n \rightarrow \infty} \sum_{k}\left|X\left(I_{k}^{n}, \omega\right)\right|^{2}
$$

always exists if $\left(I_{k}^{n}\right), k=1,2, \cdots, k_{n}$, are partitions of the $\lambda$-axis, the $(n+1)$ st one refining the $n$ th.

8. The growth of $Z(t, \omega)$.

Theorem 7. Let $X_{1}, X_{2}, \cdots$ satisfy $\left(\mathrm{A}_{1-3}\right)$ and let $\delta>0$. Then for almost all $\omega$

$$
Z_{*}(n \delta, \omega)=o(\lg n)^{1 / 2}, \quad n \rightarrow \infty
$$




\section{If moreover}

$$
\sum b_{n}\left|\lambda_{n}\right|^{2 \alpha}<\infty
$$

for some $\alpha>0$, then

$$
Z_{*}(t, \omega)=o(\lg t)^{1 / 2}, \quad t \rightarrow \infty .
$$

It is possible to replace (54) by $\sum b_{n}\left(\lg \left|\lambda_{n}\right|\right)^{1+\bullet}<\infty$, but the proof becomes much more involved.

In order to prove (53) we note that for almost all $\omega$ and for all $\rho$

$$
\sup _{n} \frac{1}{n} \sum_{1}^{n} A_{\rho}\left\{Z_{*}(k \delta, \omega)\right\}<\infty .
$$

(The proof is similar to that of (41).) Hence

$$
A_{\rho}\left\{Z_{*}(n \delta, \omega)\right\}<C n-1=C(\omega, \rho) n-1,
$$

or

$$
\exp \left\{\rho Z_{*}^{2}(n \delta, \omega)\right\}<C n, Z_{*}^{2}(n \delta, \omega)<\frac{1}{\rho}(\lg n+\lg C) .
$$

Since $\rho$ may be chosen at will, (53) is true.

When $\alpha=1$ the proof of (55) is quite easy. Let us drop $\omega$ from the notation, as what we say will be true for almost every $\omega$, and define

$$
Y_{k}(t)=\frac{d Z_{k}(t)}{d t}=i \sum_{j=1}^{k} \lambda_{j} X_{j} e^{i \lambda_{j} t}
$$

Then

$$
\begin{aligned}
& Z_{k}(t)=Z_{k}(n)+\int_{n}^{t} Y_{k}(t) d t, \quad n \leqq t<n+1, \\
& Z_{*}(t) \leqq Z_{*}(n)+\int_{n}^{n+1} Y_{*}(t) d t, \quad n \leqq t<n+1 .
\end{aligned}
$$

The first term on the right in $(57)$ is $o(\lg t)^{1 / 2}$. As for the second term, Lemma 10 applied to the random variables $\lambda_{j} X_{j}$, which satisfy $\left(\mathrm{A}_{1-3}\right)$ if $\alpha=1$, yields

$$
\int_{0}^{n} A_{\rho}\left\{Y_{*}(t)\right\} d t<C n-1=C(\omega, \rho) n-1,
$$

or

$$
\int_{n}^{n+1} \exp \left\{\rho Y_{*}^{2}(t)\right\} d t<C n
$$

Since $\exp \left(\rho x^{2}\right)$ is convex, 


$$
\begin{aligned}
\exp \left\{\rho\left(\int_{n}^{n+1} Y_{*}(t) d t\right)^{2}\right\} & \leqq \int_{n}^{n+1} \exp \left\{\rho Y_{*}^{2}(t)\right\} d t \\
& \leqq C n, \\
\left(\int_{n}^{n+1} Y_{*}(t) d t\right)^{2} & \leqq \frac{1}{\rho}(\lg n+\lg C),
\end{aligned}
$$

and the second term on the right in (57) is $o(\lg t)^{1 / 2}$.

According to what has just been proved, it suffices to consider only series with $\lambda_{k}>1$ when $0<\alpha<1$. As in $\S 5$ it is convenient to change our point of view and prove the following statement, which (together with what has already been shown) is equivalent to Theorem $7:$ Let $X_{1}, X_{2}, \cdots$ satisfy $\left(\mathrm{A}_{1-3}\right)$ and let $\lambda_{k}>1$. Then

$$
Z_{*}^{\alpha}(t)=\sup _{\boldsymbol{k}}\left|\sum_{j=1}^{k} \frac{X_{j}}{\left(i \lambda_{j}\right)^{\alpha}} e^{i \lambda_{j} t}\right|=o(\lg t)^{1 / 2} .
$$

Just as in (39),

$$
\begin{aligned}
Z_{k}^{\alpha}(t) & =\sum_{1}^{k}\left(i \lambda_{j}\right)^{-\alpha} X_{j} e^{i \lambda t} \\
& =\frac{1}{\Gamma(\alpha)} \int_{0}^{\delta} u^{\alpha-1} Z_{k}(t-u) d u+\sum_{1}^{k}\left(i \lambda_{j}\right)^{-1} F_{\delta}\left(\lambda_{j}\right) X_{j} e^{i \lambda_{j} t}, \\
Z_{*}^{\alpha}(t) & \leqq \frac{1}{\Gamma(\alpha)} \int_{0}^{\delta} u^{\alpha-1} Z_{*}(t-u) d u+\sup _{\boldsymbol{k}}\left|\sum_{1}^{k}\left(i \lambda_{j}\right)^{-1} F_{\delta}\left(\lambda_{j}\right) X_{j} e^{i \lambda_{j} t}\right|
\end{aligned}
$$

where $F_{\delta}(\lambda)$ is bounded in $\lambda \geqq 1$ for any choice of $\delta>0$. The second term on the right is $o(\lg t)^{1 / 2}$ since the random variables $\left(i \lambda_{j}\right)^{-1} F_{\delta}\left(\lambda_{j}\right) X_{j}$ satisfy the hypotheses of Theorem 7 with $\alpha=1$. We need a lemma to deal with the first term.

LEMMA 16. If $g(x) \geqq 0$ then

$$
\left\{\int_{0}^{1} g^{p} d x\right\}^{1 / p} \leqq K_{p}+\left(\lg \int_{0}^{1} \exp g^{2} d x\right)^{1 / 2} .
$$

The function $\exp u^{2 / p}$ is convex for $u^{2 / p}>\operatorname{Max}(0, p / 2-1)$. When $g^{2} \geqq p / 2$ -1 throughout $0<x<1$, we replace $u$ by $g^{p}$ and use Jensen's inequality:

$$
\exp \left(\int g^{p} d x\right)^{2 / p} \leqq \int \exp g^{2} d x,
$$

which amounts to (59) with $K_{p}=0$. If $g^{2}$ is sometimes less than $p / 2-1$, replace $g^{2}$ by $\operatorname{Max}\left(g^{2}, p / 2-1\right)$ in (60). The result is (59). Clearly (59) is true if $\int_{0}^{1}$ is replaced by $\delta^{-1} \int_{0}^{\delta}$. 
We use Hölder's inequality with exponents $2 / \alpha$ and $2 /(2-\alpha)$, then Lemma 16:

$$
\begin{aligned}
& \int_{0}^{\delta} u^{\alpha-1} Z_{*}(t-u) d u \\
& =\delta \rho^{-1 / 2} \frac{1}{\delta} \int_{0}^{\delta} u^{\alpha-1} \rho^{1 / 2} Z_{*}(t-u) d u \\
& \leqq \delta \rho^{-1 / 2}\left\{\frac{1}{\delta} \int_{0}^{\delta} u^{(2 \alpha-2) /(2-\alpha)} d u\right\}^{1-\alpha / 2}\left\{\frac{1}{\delta} \int_{0}^{\delta}\left(\rho^{1 / 2} Z_{*}(t-u)\right)^{2 / \alpha} d u\right\}^{\alpha / 2} \\
& \leqq \delta^{\alpha / 2} \rho^{-1 / 2} L_{\alpha}+\delta \rho^{-1 / 2} L_{\alpha}\left\{\lg \frac{1}{\delta} \int_{0}^{\delta} \exp \left(\rho Z_{*}^{2}(t-u)\right) d u\right\}^{1 / 2} .
\end{aligned}
$$

Here $L_{\alpha}$ can be majorized (for $\delta<1$ ) by

$$
L_{\alpha}<\left\{1+\int_{0}^{1} u^{(2 \alpha-2) /(2-\alpha)} d u\right\}\left(K_{2 / \alpha}+1\right)
$$

Also

$$
\int_{0}^{\delta} \exp \left(\rho Z_{*}^{2}(t-u)\right) d u \leqq \int_{0}^{1} \exp \left(\rho Z_{*}^{2}(t-u)\right) d u \leqq C(\rho, \omega) \mid t^{\prime}
$$

by an argument like that leading to (58). Hence (61) implies that if $\eta>0$, then $\delta$ can be chosen so small that

$$
\int_{0}^{\delta} u^{\alpha-1} Z_{*}(t-u) d u<\eta(\lg t)^{1 / 2}
$$

Thus $Z_{*}^{\alpha}(t)=o(\lg t)^{1 / 2}$ and the theorem is proved.

TheOREM 8. Let $X(I, \omega)$ satisfy $\left(B_{1-4}\right)$ and let $\delta>0$. Then for almost all $\omega$ there is $a C=C(\omega)$ such that

$$
Z_{*}(n \delta, \omega)<C(\lg n)^{1 / 2},
$$$$
n \rightarrow \infty \text {. }
$$

If also

$$
\int_{-\infty}^{\infty}|\lambda|^{2 \alpha} B(d \lambda)<\infty
$$

then there is a $\bar{C}=\bar{C}(\omega)$ such that

$$
Z_{*}(t, \omega) \leqq \bar{C}(\lg t)^{1 / 2},
$$$$
t \rightarrow \infty
$$

The proof is like that of Theorem 7 except that we may not take $\rho$ arbitrarily large.

9. An example. A stochastic process $Z(t, \omega)$ is called strictly stationary if 
for every collection $E_{1}, \cdots, E_{n}$ of open sets of complex numbers and every set $t_{1}, \cdots, t_{n}$ the probability

$$
P\left\{Z\left(t_{j}+s\right) \in E_{j}, j=1, \cdots, n\right\}
$$

is independent of $s$. According to Blanc-Lapierre and Fortet [1], when the spectrum of $Z(t)$ is a discrete set $\left(\lambda_{k}\right)$ and the $\lambda_{k}$ are linearly independent over the field of rationals, one can write

$$
Z(t, \omega)=\sum X_{k}(\omega) U_{k}(\omega) e^{i \lambda_{k} t},
$$

where the $X_{k}$ are independent among themselves and the $X_{k}$ are independent of the $U_{j}$. Moreover each $X_{k}$ is uniformly distributed on the unit circle $|x|$ $=1$. These facts are easy to prove.

It can also be shown that $\sum\left|U_{k}(\omega)\right|^{2}<\infty$ for almost all $\omega$. It is not hard to see that all our theorems about series apply to $Z(t)$. One has only to fix the values of the $U_{k}$ and consider the conditional distribution of the $X_{k}$. The condition $\sum b_{n}\left|\lambda_{n}\right|^{\epsilon}<\infty$ is to be replaced by $\sum\left|U_{n}(\omega)\right|^{2}\left|\lambda_{n}\right|^{\epsilon}<\infty$ for almost all $\omega$.

10. Fourier-Wiener transforms. A random additive function of intervals $Y(I, \omega)$ is a Wiener process if it has independent increments and can be written $Y(I)=Y_{1}(I)+i Y_{2}(I)$, with $Y_{1}$ and $Y_{2}$ real processes, independent the one of the other, and

$$
P\left\{Y_{k}(I)<y\right\}=(\pi|I|)^{-1 / 2} \int_{-\infty}^{y} e^{-u^{2} /|I|} d u, \quad k=1,2 .
$$

Here $|I|$ is the length of $I$.

We take $\Omega$ to be the set of all additive functions of intervals which depend continuously on the end points of the interval, so that $Y(I, \omega)=\omega(I)$.

When $f(\lambda)$ belongs to $L_{-\infty, \infty}^{2}$, the integral

$$
\int_{-\infty}^{\infty} f(\lambda) Y(d \lambda)
$$

can be defined as a strong limit in $L_{\Omega}^{2}$. It is a random variable whose real and imaginary parts are independent normal variables with mean zero and vari ance

$$
\frac{1}{2}\|f\|^{2} \equiv \frac{1}{2} \int_{-\infty}^{\infty}|f(\lambda)|^{2} d \lambda
$$

The Fourier-Wiener transform of such an $f$ is

$$
Z(t)=Z(t, \omega)=\int_{-\infty}^{\infty} e^{i t \lambda} f(\lambda) Y(d \lambda, \omega) .
$$

If $g(t)$ is the Fourier transform of $f(\lambda)$, then $Z(t)$ may also be written 


$$
Z(t, \omega)=\int_{-\infty}^{\infty} g(t-s) \tilde{Y}(d s, \omega)
$$

where $\tilde{Y}(I, \omega)$ is again a Wiener process.

The auto-correlation function of $Z(t)$ is

$$
\begin{aligned}
\phi(s) & =\int_{\mathbf{\Omega}} \bar{Z}(t) Z(t+s) d \omega \\
& =\int_{-\infty}^{\infty}|f(\lambda)|^{2} e^{i s \lambda} d \lambda \\
& =\int_{-\infty}^{\infty} \bar{g}(t) g(t+s) d t .
\end{aligned}
$$

We shall use the facts above, and also the correspondence between unitary transformations of $L_{-\infty, \infty}^{2}$ and measure preserving transformations of $\Omega$ without special mention. (See Paley-Wiener [9] and Bochner [2].)

It should be clear that our theorems hold for Fourier-Wiener transforms. The only difficulty is that the $Z(t)$ in (62) is defined only almost everywhere in $\Omega \times(t)$. We may avoid this difficulty by defining

$$
X(I, \omega)=\int_{I} f(\lambda) Y(d \lambda, \omega)
$$

for intervals $I$ with rational end points as a strong limit in $L^{2}$, then extending the definition of $X$ to all intervals. The resulting function satisfies $\left(B_{1-4}\right)$ if a null set of $\Omega$ is ignored. Moreover, the $Z(t, \omega)$ of (62) is the limit (for almost all $\omega$ and $t$ ) of

$$
\int_{-\Lambda}^{\Lambda} e^{i \lambda t} X(d \lambda, \omega)
$$

as $\Lambda \rightarrow \infty$. The last integral is to be taken in the sense of $\S 4$. Our statements in this section really concern the limit of (65).

Theorem 9. If $f \in L^{2}$ and if

$$
\int_{-\infty}^{\infty}\left\{\lg \left(1+\lambda^{2}\right)\right\}^{1+\epsilon}|f(\lambda)|^{2} d \lambda<\infty,
$$

then (for almost all $\omega) Z(t, \omega)$ is continuous in $t$. If $f \in L^{2}$ and if

$$
\int_{-\infty}^{\infty}|\lambda|^{2 \alpha}|f(\lambda)|^{2} d \lambda<\infty, \quad 0<\alpha \leqq 1,
$$

or, what is the same as (66) for $0<\alpha<1$, if 


$$
\int_{0}^{\infty} \frac{2 \phi(0)-\phi(s)-\phi(-s)}{s^{1+2 \alpha}} d s<\infty,
$$

then $Z(t, \omega) \in H_{\alpha}$ for almost all $\omega$.

THEOREM 10. If $f \in L^{2}$ and if (66) or (67) is true, then for almost all $\omega$,

$$
\limsup _{t \rightarrow \infty} \frac{|Z(t, \omega)|}{(\lg t)^{1 / 2}}=\|f\| \text {. }
$$

Only Theorem 10, which sharpens Theorem 8 , needs an indication of the proof. We assume $\|f\|=1$ and prove first that (68) is true with $\leqq$ for $=$.

Clearly, for $\rho<1$,

$$
\begin{aligned}
\int_{\Omega} \exp \left\{\rho|Z(t)|^{2}\right\} d \omega & =\int_{\Omega} \exp \left\{\rho Z_{1}^{2}+\rho Z_{2}^{2}\right\} d \omega \\
& =\frac{1}{\pi} \int_{-\infty}^{\infty} \int_{-\infty}^{\infty} e^{\rho u^{2}+\rho v^{2}} e^{-u^{2}-v^{2}} d u d v<\infty .
\end{aligned}
$$

( $Z_{1}$ is the real part of $Z$, and $Z_{2}$ is the imaginary.) Since the transformations $Z(t) \rightarrow Z(t+s)$ can be regarded as the result of a measure preserving flow of $\Omega$ into itself, the maximal ergodic theorem and (69) imply

$$
\begin{aligned}
& \sup \frac{1}{n} \sum_{1}^{n} A_{\rho}\{Z(k \delta, \omega)\}<\infty, \\
& \sup \frac{1}{T} \int_{0}^{T} A_{\rho}\{Z(t, \omega)\} d t<\infty
\end{aligned}
$$

for every $\rho<1$, every $\delta>0$, and almost every $\omega$. From (70) and (71) we conclude, as in the proof of Theorem 7 , that

$$
\begin{gathered}
|Z(n \delta, \omega)|<\left\{\rho^{-1 / 2}+o(1)\right\}(\lg n \delta)^{1 / 2} \\
\frac{1}{\delta} \int_{n \delta}^{(n+1) \delta}|Z(t, \omega)| d t<\left\{\rho^{-1 / 2}+o(1)\right\}(\lg n \delta)^{1 / 2}
\end{gathered}
$$

This much is accomplished without using (66). When $\alpha=1, Z(t)$ is differentiable and we write

$$
Z(t)=Z(n \delta)+\int_{n \delta}^{t} Z^{\prime}(u) d u, \quad n \delta \leqq t<(n+1) \delta .
$$

Since $Z^{\prime}(t)$ is the Fourier-Wiener transform of $i \lambda f(\lambda)$, (73) holds with $Z$ replaced by $Z^{\prime}$ and $\rho$ replaced by any number less than $\|\lambda f(\lambda)\|$, say by $A$ $=\|\lambda f(\lambda)\| / 2$. Thus (72) and (73) imply

$$
|Z(t)|<\left\{\rho^{-1 / 2}+A^{-1 / 2} \delta+o(1)\right\}(\lg n \delta)^{1 / 2},
$$


and this is equivalent to (68) with $\leqq$ for $=$, since $\rho$ and $\delta$ are restricted only by $\rho<1$ and $\delta>0$.

When $0<\alpha<1$ we write

$$
\begin{aligned}
Z(t) & =\int_{-L}^{L} e^{i \lambda t} f(\lambda) Y(d \lambda)+\int_{|\lambda|>L} e^{i \lambda t} f(\lambda) Y(d \lambda) \\
& =Z_{3}(t)+Z_{4}(t) .
\end{aligned}
$$

By what has already been proved, (68) is true with $Z_{3}$ for $Z$ and $\leqq$ for $=$. As for $Z_{4}$, if $\eta>0$ we choose $L$ so large that

$$
\int_{|\lambda|>L}|f(\lambda)|^{2}|\lambda|^{2 \alpha} d \lambda<\eta
$$

The argument concluding the proof of Theorem 7 then shows that

$$
\left|Z_{4}(t)\right|<\left\{C \eta^{1 / 2}+o(1)\right\}(\lg t)^{1 / 2}
$$

with $C$ independent of $\eta$. So (68) is true with $\leqq$ for $=$.

In order to show that $(68)$ is true when $=$ is replaced by $\geqq$, we use the representation (63), in which $g(t)$ is the Fourier transform of $f(\lambda)$. A few preliminary remarks are needed.

It is well known that if $h(\lambda) \in L^{2}$ and $p(\lambda) \in L$, then $\int h(\lambda-\mu) p(\mu) d \mu$ belongs to $L^{2}$.

Suppose that $f(\lambda)$ and $|\lambda| \alpha f(\lambda)$ belong to $L^{2}$, that $g(t)$ is the Fourier transform of $f(\lambda)$, and that $q(t)$ is an infinitely differentiable function which vanishes outside some bounded interval. The inverse Fourier transform of $q(t) g(t)$ is

$$
(2 \pi)^{1 / 2} \int_{-\infty}^{\infty} f(\lambda-\mu) p(\mu) d \mu,
$$

where $p(\lambda)$ is the inverse Fourier transform of $q(t)$. It is clear that $p(\lambda)$ vanishes at infinity faster than any power of $\lambda$, so that $p(\lambda)$ and $|\lambda|{ }^{\alpha} p(\lambda)$ both belong to $L$. Noting that $|\lambda|^{\alpha} \leqq|\lambda-\mu|^{\alpha}+|\mu|^{\alpha}$ for $0<\alpha \leqq 1$, we have

$$
\begin{aligned}
|\lambda|^{\alpha}\left|\int_{-\infty}^{\infty} f(\lambda-\mu) p(\mu) d u\right| \leqq & \int_{-\infty}^{\infty}|\lambda-\mu|^{\alpha}|f(\lambda-\mu)||p(\mu)| d \mu \\
& +\int_{-\infty}^{\infty}|f(\lambda-\mu)||\mu|^{\alpha}|p(\mu)| d \mu
\end{aligned}
$$

and by the first remark, both terms on the right belong to $L^{2}$. Hence $q(t) g(t)$ is the Fourier transform of a function satisfying (66). The same is true of $(1-q(t)) g(t)$.

We take for $q(t)$ a function which vanishes outside $(-L, L)$, equals 1 in $(1-L, L-1)$, and elsewhere assumes only values between 0 and 1 . Then 


$$
\begin{aligned}
Z(t) & =\int_{-\infty}^{\infty} q(s) g(s) \tilde{Y}(t-d s)+\int_{-\infty}^{\infty}(1-q(s)) g(s) \tilde{Y}(t-d s) \\
& =Z_{5}(t)+Z_{6}(t) .
\end{aligned}
$$

If $L$ is so large that $\|(1-q) g\|<\eta$ then

$$
\limsup _{t \rightarrow \infty} \frac{\left|Z_{6}(t)\right|}{(\lg t)^{1 / 2}} \leqq \eta
$$

On the other hand, the random variables $Z_{5}(0), Z_{5}(2 L), Z_{5}(4 L), \cdots$ are independent and for large $z$

$$
\begin{aligned}
P\left\{\left|Z_{5}(2 n L)\right|>z\right\} & \geqq P\left\{\text { real part of } Z_{5}(2 n L)>z\right\} \\
& \geqq \frac{1}{\sigma \pi^{1 / 2}} \int_{z}^{\infty} e^{-u^{2} / \sigma^{2}} d u \\
& >\frac{\sigma}{3 z \pi^{1 / 2}} e^{-z^{2} / \sigma^{2}} .
\end{aligned}
$$

Here $\sigma=\|q g\|$. Thus the series

$$
\sum_{n=1}^{\infty} P\left\{\left|Z_{5}(2 n L)\right|>\sigma(\lg 2 n L)^{1 / 2}\right\}
$$

diverges. Accordingly, for almost all $\omega$

$$
\left|Z_{5}(2 n L)\right|>\sigma(\lg 2 n L)^{1 / 2}
$$

for infinitely many $n$. Since $\eta$ can be chosen small and $\sigma$ can be chosen close to 1 , it follows from (75) and (77) that (68) is true with $\geqq$ for $=$. This completes the proof of Theorem 10.

The limit in (68) remains the same if $Z(t)$ is restricted to a sector or if the argument of $Z(t)$ is restricted by

$$
\text { - }(\lg t)^{-1 / 2}<\arg Z(t)<(\lg t)^{-1 / 2},
$$

for the series corresponding to (76) diverges.

It is easy to see that (66) may be dispensed with if $t$ is restricted to an arithmetical progression:

THEOREM $10^{\prime}$. If $f \in L^{2}$, then for amost every $\omega$

$$
\limsup _{n \rightarrow \infty} \frac{|Z(n)|}{\lg ^{1 / 2} n}=\|f\| \text {. }
$$

A finer study of the properties of $Z(t)$ requires more knowledge of $f(\lambda)$ than (66) furnishes. We give one example, which by no means exhausts the subject. 
If $I=(a, b)$ we write $c I$ for $(c a, c b)$ and interpret $Y(c d \lambda)$ as the differential of $Y(c I)$. Let us define

$$
W(u)=\int_{0}^{\infty}\left(e^{i \lambda}-1\right) \lambda^{\beta-1} e^{u / 2} Y\left(e^{-u} d \lambda\right), \quad-\frac{1}{2}<\beta<\frac{1}{2} .
$$

By making use of the correspondence between unitary transformations of $L^{2}$ and measure preserving transformations of $\Omega$, it is not difficult to show that $W$ is a Fourier-Wiener transform, though of course not a transform of the $Y$ appearing in (78).

In order to apply Theorem 10 , we verify that condition (67) is satisfied. The auto-correlation function is

$$
\phi(v)=e^{(\beta-1 / 2) v} \int_{0}^{\infty}\left(e^{-i \lambda}-1\right)\left(e^{i \lambda e^{v}}-1\right) \lambda^{2 \beta-2} d \lambda .
$$

We have

$$
\phi(0)=\int_{0}^{\infty}\left|e^{i \lambda}-1\right|^{2} \lambda^{2 \beta-2} d \lambda=\frac{2 \pi \sin \beta \pi}{\Gamma(2-2 \beta) \sin 2 \beta \pi}=K_{\beta}^{2}
$$

and

$$
\int_{0}^{\infty}\left|\left(e^{i \lambda(1+\gamma)}-1\right) \lambda^{\beta-1}-\left(e^{i \lambda}-1\right) \lambda^{\beta-1}\right|^{2} d \lambda=K_{\beta}^{2} \gamma^{1-2 \beta} .
$$

Hence, by the inequality of Cauchy-Schwarz,

$$
\begin{aligned}
\phi(0)-\phi(v) & =\left(1-e^{(\beta-1 / 2) v}\right) \phi(0)+e^{(\beta-1 / 2) v} \int_{0}^{\infty}\left(e^{-i \lambda}-1\right)\left(e^{i \lambda}-e^{i \lambda} e^{v}\right) \lambda^{2 \beta-2} d \lambda \\
& =O\left(v^{1 / 2-\beta}\right) .
\end{aligned}
$$

This is enough to show that (67) holds for some $\alpha>0$, for $1 / 2-\beta>0$. Thus, for almost all $\omega$,

$$
\limsup _{|u| \rightarrow \infty} \frac{|W(u)|}{(\lg |u|)^{1 / 2}}=K_{\beta}
$$

In order to use (79) we need a lemma, whose proof is left to the reader.

Lемма 17. Suppose that $|f(\lambda)| \geqq|g(\lambda)|$, that

$$
\begin{aligned}
& U(t, \omega)=\int h(\lambda, t) f(\lambda) Y(d \lambda, \omega), \\
& V(t, \omega)=\int h(\lambda, t) g(\lambda) Y(d \lambda, \omega),
\end{aligned}
$$


and that

$$
\lim \sup |V(t, \omega)| \geqq 1
$$

for almost all $\omega$. Then also

$$
\lim \sup |U(t, \omega)| \geqq 1
$$

for almost all $\omega$.

Note that for $t>0$,

$$
\begin{aligned}
V(t) & \equiv \int_{0}^{\infty}\left(e^{i \lambda t}-1\right) \lambda^{\beta-1} Y(d \lambda) \\
& =t^{1 / 2-\beta} W(\lg t),
\end{aligned}
$$

so that

$$
\lim \sup \frac{|V(t)|}{t^{1 / 2-\beta}(\lg |\lg t|)^{1 / 2}}=K_{\beta}
$$

as $t \rightarrow \infty$ or $t \rightarrow 0$, for almost all $\omega$. This together with the lemma yields the following statement: If

$$
C_{1} \lambda^{\beta-1} \leqq f(\lambda) \leqq C_{2} \lambda^{\beta-1}, \quad-\frac{1}{2}<\beta<\frac{1}{2},
$$

and

$$
Z(t)=\int_{0}^{\infty}\left(e^{i \lambda t}-1\right) f(\lambda) Y(d \lambda)
$$

then for almost all $\omega$

$$
C_{1} K_{\beta} \leqq \lim \sup \frac{|Z(t)|}{t^{1 / 2-\beta}(\lg |\lg t|)^{1 / 2}} \leqq C_{2} K_{\beta}, \quad t \rightarrow \infty \text { or } t \rightarrow 0 .
$$

An $f$ satisfying the above inequalities cannot possibly belong to $L^{2}$. We shall see that (82) really corresponds to two theorems concerning Fourier-Wiener transforms-one dealing with local behavior and one with averages.

THEOREM 11. If $f \in L^{2}$, if (81) is true for large $\lambda$, and if

$$
Z(t)=\int_{0}^{\infty} f(\lambda) e^{i \lambda t} Y(d \lambda)
$$

then for every $t$ and almost all $\omega$,

$$
C_{1} K_{\beta} \leqq \lim _{|h| \rightarrow 0} \frac{|Z(t+h)-Z(t)|}{|h|^{1 / 2-\beta}(\lg \lg 1 /|h|)^{1 / 2}} \leqq C_{2} K_{\beta}
$$


THEOREM 12. If $f \in L^{2}$, if $C_{1} \lambda^{\beta} \leqq f(\lambda) \leqq C_{2} \lambda^{\beta}$ for small $\lambda$ with $-1 / 2<\beta<1 / 2$, and if $Z(t)$ is again defined by (83), then

$$
C_{1} K_{\beta} \leqq \limsup _{T \rightarrow \infty} \frac{\left|\int_{0}^{T} Z(t) d t\right|}{T^{1 / 2-\beta}(\lg \lg T)} \leqq C_{2} K_{\beta}
$$

for almost all $\omega$.

To prove Theorem 11 we make the following observations: $Z(t)$ is stationary, so it suffices to consider $Z(t)-Z(0)$ as $t \searrow 0$. Now, the local behavior of $Z(t)$ depends, as we know, only on the part $\int_{\vec{b}}^{\infty}$, for $\int_{0}^{L}$ is an analytic function for every $\omega$. Since the same is true of $V(t)$ as defined by $(80)$, we compare

$$
\int_{L}^{\infty}\left(e^{i \lambda t}-1\right) \lambda^{\beta-1} Y(d \lambda)
$$

with

$$
\int_{L}^{\infty}\left(e^{i \lambda t}-1\right) f(\lambda) Y(d \lambda)
$$

and use Lemma 17 to prove the theorem.

The proof of Theorem 12 is similar. We have

$$
\int_{0}^{T} Z(t) d t=-i \int_{0}^{L}\left(e^{i \lambda T}-1\right) f(\lambda) \lambda^{-1} Y(d \lambda)-i \int_{L}^{\infty}\left(e^{i \lambda T}-1\right) f(\lambda) \lambda^{-1} Y(d \lambda) .
$$

The second integral is $O(\lg T)^{1 / 2}$ by Theorem 10 , and we compare the first integral with

$$
\int_{0}^{L}\left(e^{i \lambda T}-1\right) \lambda^{\beta-1} Y(d \lambda) .
$$

If one makes assumptions only about the behavior of

$$
\int \lambda^{-2} \sin ^{2} \frac{\lambda t}{2}|f(\lambda)|^{2} d \lambda
$$

results less precise than (84) and (85) can be proved.

Theorems 11 and 12 contain the laws of the iterated logarithm for Brownian motion. One has only to consider (80) written for $\beta=0$,

$$
V(t)=\int_{0}^{\infty} \frac{e^{i \lambda t}-1}{\lambda} Y(d \lambda)
$$

This is the complex (two-dimensional) Brownian motion which starts from the origin at $t=0$. For $t \rightarrow \infty$ the limit following (80) gives the law of the 
iterated logarithm at $\infty$; for $t \rightarrow 0$, the local law of the iterated logarithm. The more refined theorems concerning upper and lower sequences escape our methods.

Theorem 4 applied to Brownian motion yields only a uniform Lipschitz condition of order $\alpha$ for every $\alpha<1 / 2$, which is somewhat weaker than what can be proved by a direct probability argument. Actually the following theorem is true for Fourier-Wiener transforms.

THEOREM 13. Under the hypotheses of Theorem $11, Z$ belongs to $H_{1 / 2-\beta}$ but not to $H_{\alpha}$ for any $\alpha>1 / 2-\beta$. (It is assumed that $C_{1}>0$ in (81).)

\section{REFERENCES}

1. A. Blanc-Lapierre and R. Fortet, C. R. Acad. Sci. Paris vol. 222 (1944) pp. 1155-1157.

2. S. Bochner, Ann. of Math. vol. 48 (1947) pp. 1014-1061.

3. J. L. Doob, Trans. Amer. Math. Soc. vol. 42 (1937) pp. 107-140.

4. W. Feller, Trans. Amer. Math. Soc. vol. 54 (1943) pp. 373-402.

5. B. Jessen, Acta Math. vol. 63 (1934) pp. 249-323.

6. J. Khintchine, Math. Zeit. vol. 18 (1923) pp. 109-116.

7. A. Kolmogorov, Math. Ann. vol. 99 (1928) pp. 309-319.

8. J. Marcinkiewicz and A. Zygmund, Studia Mathematica vol. 7 (1938) pp. 104-120.

9. R. E. A. C. Paley and N. Wiener, Fourier transforms in the complex domain, Amer. Math. Soc. Colloquium Publications, vol. 19, New York, 1934.

10. R. E. A. C. Paley and A. Zygmund, Proc. Cambridge Philos. Soc. vol. 26 (1930) pp. 337-357, 458-474; and vol. 28 (1932) pp. 190-205.

11. A. Zygmund, Trigonometrical series, Warsaw, 1935.

CORNELL UNIVERSITY

ITHACA, N. Y. 